\title{
Embryonal tumors with abundant neuropil and true rosettes: 2 illustrative cases and a review of the literature
}

\author{
Sunil Manjila, M.D., ${ }^{1}$ Abhishek Ray, M.D., ${ }^{1}$ Yin Hu, M.D., ${ }^{1}$ Dan X. CaI, M.D., Ph.D., ${ }^{3}$ \\ Mark L. Cohen, M.D., ${ }^{2}$ and Alan R. Cohen, M.D. ${ }^{1}$ \\ ${ }^{1}$ Division of Pediatric Neurosurgery, Rainbow Babies and Children's Hospital; ${ }^{2}$ Division of \\ Neuropathology, The Neurological Institute, University Hospital Case Medical Center, Case Western Reserve \\ University School of Medicine; and ${ }^{3}$ Department of Pathology, MetroHealth Medical Center, Cleveland, Ohio
}

\begin{abstract}
Embryonal tumor with abundant neuropil and true rosettes (ETANTR) is a recently identified variant of primitive neuroectodermal tumor, with fewer than 50 cases reported in the literature to date. Histologically, this tumor has features of ependymoblastoma and neuroblastoma, demonstrating areas of fine fibrillary neuropil intermingled with ependymoblastic rosettes and zones of undifferentiated neuroepithelial cells. However, ETANTR is distinguished pathologically from other embryonal tumors by the striking abundance of neuropil. Clinically, ETANTRs have shown high malignant potential and poor clinical outcome despite aggressive treatment. The authors describe 2 illustrative surgical cases of ETANTR, one involving the longest reported survival in the literature to date. The other had a poor outcome despite high-dose adjuvant chemotherapy with sequential autologous hematopoietic stem cell rescue. The authors review the natural history and treatment strategies available for this unusual malignant pediatric brain tumor. (DOI: 10.3171/2010.10.FOCUS10226)
\end{abstract}

KEY WORDS $\bullet$ embryonal tumor with abundant neuropil and true rosettes $\bullet$
primitive neuroectodermal tumor $\bullet$ WHO classification $\bullet$ neuropil
ependymoblastoma $\bullet \quad$ brain tumor

$\mathrm{E}$ MBRYONAL tumor with abundant neuropil and true rosettes (ETANTR) has been identified as a histologically distinctive CNS PNET. It has mixed histological features of ependymoblastoma and neuroblastoma. This new subtype was first reported in 2000 by Eberhart and colleagues. ${ }^{6}$ The most distinctive and consistent features of ETANTR are the presence of undifferentiated neuroepithelial cells and ependymoblastic rosettes arising abruptly in a paucicellular background of abundant neoplastic neuropil.

The classification of CNS PNETs has long been controversial. In 2007, the WHO classified CNS PNETs as one of 3 histological subtypes of primitive embryonal tumors, the other 2 subtypes being medulloblastoma and atypical teratoid/rhabdoid tumor (AT/RT). ${ }^{14}$ The WHO recognizes 5 PNET subtypes including PNET not otherwise specified (NOS), medulloepithelioma, neuroblastoma, ganglioneuroblastoma, and ependymoblastoma. ETANTR appears similar to ependymoblastoma and neuroblastoma but has unique histological, immunohistochemical, and ultrastructural features. The WHO classifies ependymoblastoma and neuroblastoma as separate

Abbreviations used in this paper: ETANTR = embryonal tumor with abundant neuropil and true rosettes; PNET = primitive neuroectodermal tumor.

entities, while ETANTR is currently considered only a variant.${ }^{14}$ Whether ETANTR should be split off as a distinct pathological entity is a topic currently under debate.

ETANTR is associated with a high potential for malignancy and a dismal prognosis. The paucity of reported cases and lack of treatment guidelines prompted us to explore the pathology and management of this unusual lesion.

Case 1

\section{Case Reports}

History and Presentation. This 4-year-old boy presented with right-sided headaches that became progressively worse over 3 weeks. The headaches became severe 4 days prior to admission and awakened him from sleep in the morning. Examination was remarkable for macrocrania and no lateralizing neurological deficits.

Imaging Evaluation. Cranial CT showed a large intraaxial right parietal brain tumor with slight calcification and significant mass effect. Magnetic resonance imaging delineated the intraaxial tumor, which was hypointense on T1-weighted images and heterogeneous on T2-weighted images, with prominent vessels within the mass. There was minimal contrast enhancement (Fig. 1). 
Operation. The patient underwent right parietal craniotomy and radical resection of the tumor. The tumor presented at the cortical surface and was adherent to the dura in some areas. It appeared as a fleshy, reddish-gray intraaxial mass that was highly vascular. Thrombosed vessels were observed within the mass. Medially, the tumor extended to the sagittal sinus and falx cerebri, which were visualized at the end of the resection. A radical tumor resection was achieved.

Postoperative Course and Findings. Postoperatively the patient had no focal neurological deficits. The biopsy showed ETANTR, strongly positive for neuropil and synaptophysin (Fig. 2). The pathological diagnosis was confirmed by external review. Postoperative MR imaging confirmed radical resection of the tumor (Fig. 3). An MR imaging study of the spine showed no evidence of disseminated disease. Cerebrospinal fluid cytology was negative for tumor.

Adjuvant Therapy and Follow-Up. The patient was treated with radiation and chemotherapy at Mott Children's Hospital, University of Michigan. Craniospinal radiation was administered over 6 weeks, with 36 Gy to the brain and spinal cord and a boost to 55.8 Gy to the tumor bed. Chemotherapy was administered according to Children's Cancer Group Protocol 99701, although the patient was treated off-study. This was a Phase I trial of the use of carboplatin as a radiation sensitizer. The patient received carboplatin at the maximum tolerated dose (cumulative dose $1050 \mathrm{mg} / \mathrm{m}^{2}$ ) and vincristine concurrently with radiation therapy. This was followed by cis-platinum (cumulative dose $450 \mathrm{mg} / \mathrm{m}^{2}$ ), vincristine (cumulative dose $30 \mathrm{mg} / \mathrm{m}^{2}$ ), and cyclophosphamide (cumulative dose 11.5 $\mathrm{mg} / \mathrm{m}^{2}$ ) over a period of 6 months (Arm B). Surveillance MR imaging was performed 4 times a year for 1 year, then 3 times a year for 1 year, then twice a year for 1 year, and then yearly thereafter. Follow-up MR imaging of the brain 7 years after surgery showed no evidence of recurrent tumor (Fig. 4). Spine imaging was performed serially during the first 4 postoperative years but not thereafter. No spinal disease was noted.

Seven years following surgery, the patient remains neurologically intact except for mild high-pitched hearing loss in the right ear. He is mainstreamed (in regular classes) in the fifth grade. He has some cognitive delay and receives assistance in mathematics. He is active physically and plays baseball and tackle football. He recently completed a 50-mile bicycle ride. He does have short stature and hypothyroidism for which he receives treatment with growth hormone and levothyroxine.

\section{Case 2}

History and Presentation. This 17-month-old previously healthy boy presented with a 3-day history of progressive lethargy, vomiting, and left-sided weakness affecting his upper extremity followed by his lower extremity, which proceeded to an acute deterioration of mental status with extensor posturing.

Imaging Evaluation. Cranial CT showed a 7-cm- diameter intraaxial lesion in the right hemisphere with edema and $12 \mathrm{~mm}$ of midline shift. Magnetic resonance imaging showed a prominent, globoid, weakly enhancing, partially cystic mass, with scattered foci of nodularity. The mass was hypointense on T1-weighted sequences. It was located in the suprasylvian region and occupied a substantial portion of the right hemisphere (Fig. 5).

First Operation, Postoperative Course, and Adjuvant Therapy. The patient underwent an emergent craniotomy and radical resection of the tumor. Postoperative MR imaging showed no significant residual tumor (Fig. 6). Pathological specimens showed ETANTR. Postoperatively, the patient did well following a course of rehabilitation. He was able to ambulate independently with a steady gait. His hemiparesis improved but did not resolve. He received adjuvant chemotherapy with cyclophosphamide, etoposide, and methotrexate.

Second Operation, Postoperative Course, and Adjuvant Therapy. Four months after surgery, he developed irritability and had frequent falls. Repeat MR imaging showed recurrence of tumor within the operative bed (Fig. 7). The patient underwent repeat tumor resection, and postoperative MR imaging again showed no residual tumor. He was started on myeloablative chemotherapy using thiotepa and carboplatin, with autologous stem cell rescue. There was initial regression of tumor on MR imaging after completion of 2 cycles of stem cell rescue, and he subsequently received a third infusion of autologous stem cells. He developed severe leukopenia and bacteremia with Klebsiella, Pseudomonas, Acinetobacter, and Enterobacter that responded to antibiotic therapy. Repeat MR imaging 1 year following the initial surgery showed a new right occipital metastatic lesion adjacent to the transverse sinus. Palliative intensity modulation radiation therapy was recommended, but the family declined further treatment and the child died shortly thereafter (Fig. 8).

\section{Discussion}

Embryonal tumor with abundant neuropil and true rosettes (ETANTR) is a histologically distinctive malignant brain tumor of childhood. Since its initial description a decade ago, there have been fewer than 50 cases cited in the literature. In the present report, we describe 2 children with supratentorial ETANTR who each presented with a short history and rapid progression of clinical symptoms. In each case, the tumor was very large and exhibited mass effect. The patient in Case 1 was treated with radical surgery, craniospinal radiation, and chemotherapy, and he is alive without clinical or radiological evidence of active disease 7 years after presentation. This represents the longest reported survival for a patient with ETANTR to date.

The explanation for this long survival is not entirely clear. The tumor was hemispheric, and we were able to achieve a radical resection. Adjuvant therapy included both craniospinal radiation and chemotherapy. The PNETs as a group show better response to radiation than chemotherapy. The use of high-dose carboplatin as a radiation sensitizer may have played a role in achieving disease con- 

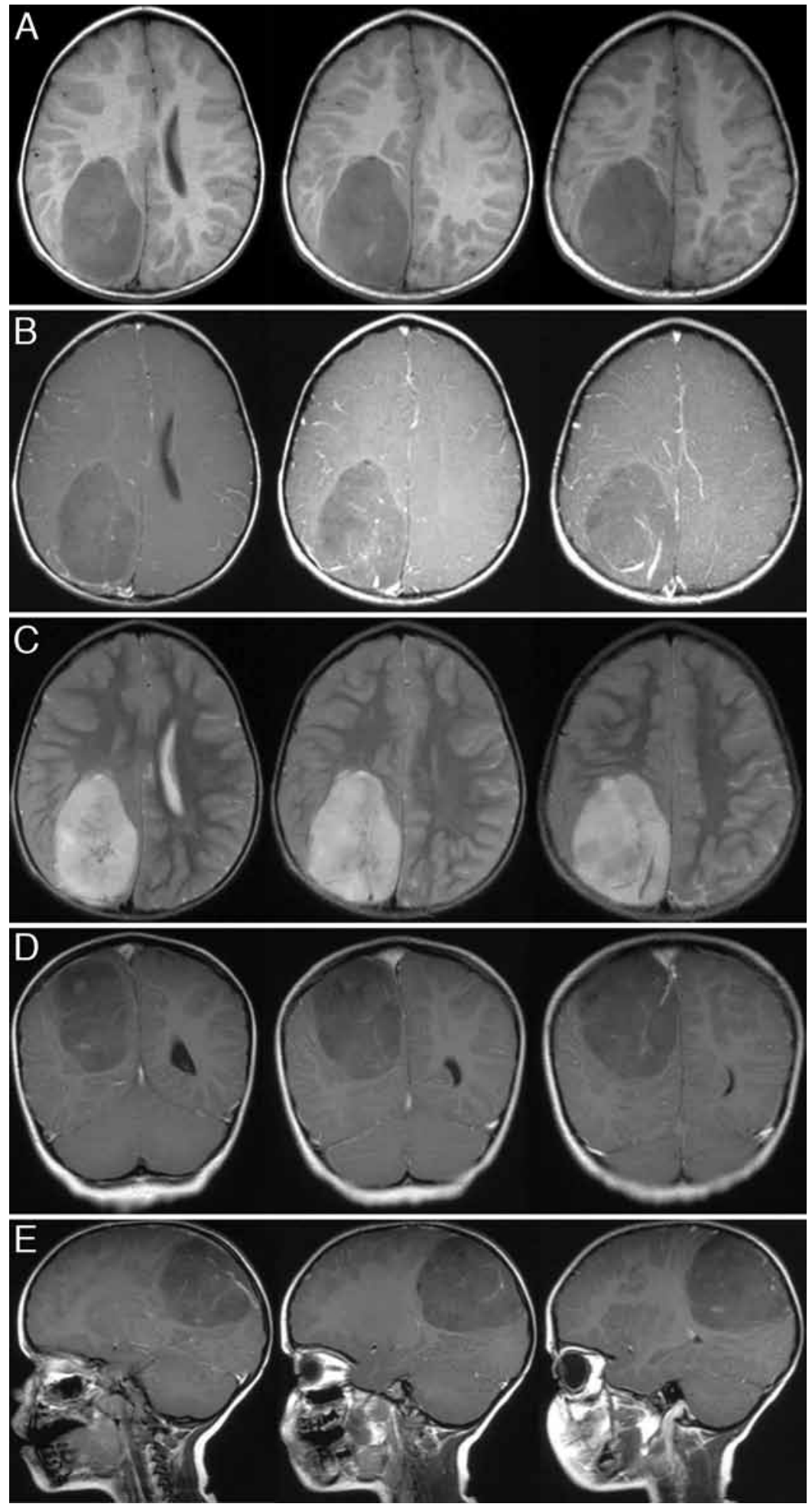

FIG. 1. Case 1. Preoperative MR imaging. A: Axial T1-weighted images obtained without contrast enhancement. B: Axial T1-weighted Gd-enhanced images. C: Axial T2-weighted images. D: Coronal Gd-enhanced T1-weighted images. E: Sagittal Gd-enhanced T1-weighted images.

trol. Other long-term survivors may exist, but, since most reported series are in pathology journals, this information is not easily available.

ETANTR was first described by Eberhart and col- leagues ${ }^{6}$ in 2000 , when they reported 7 cases as a distinctive form of CNS PNET. They initially called the lesion "pediatric neuroblastic tumor with abundant neuropil and true rosettes" (PNTANTR). ${ }^{7,8}$ The finding of micro- 


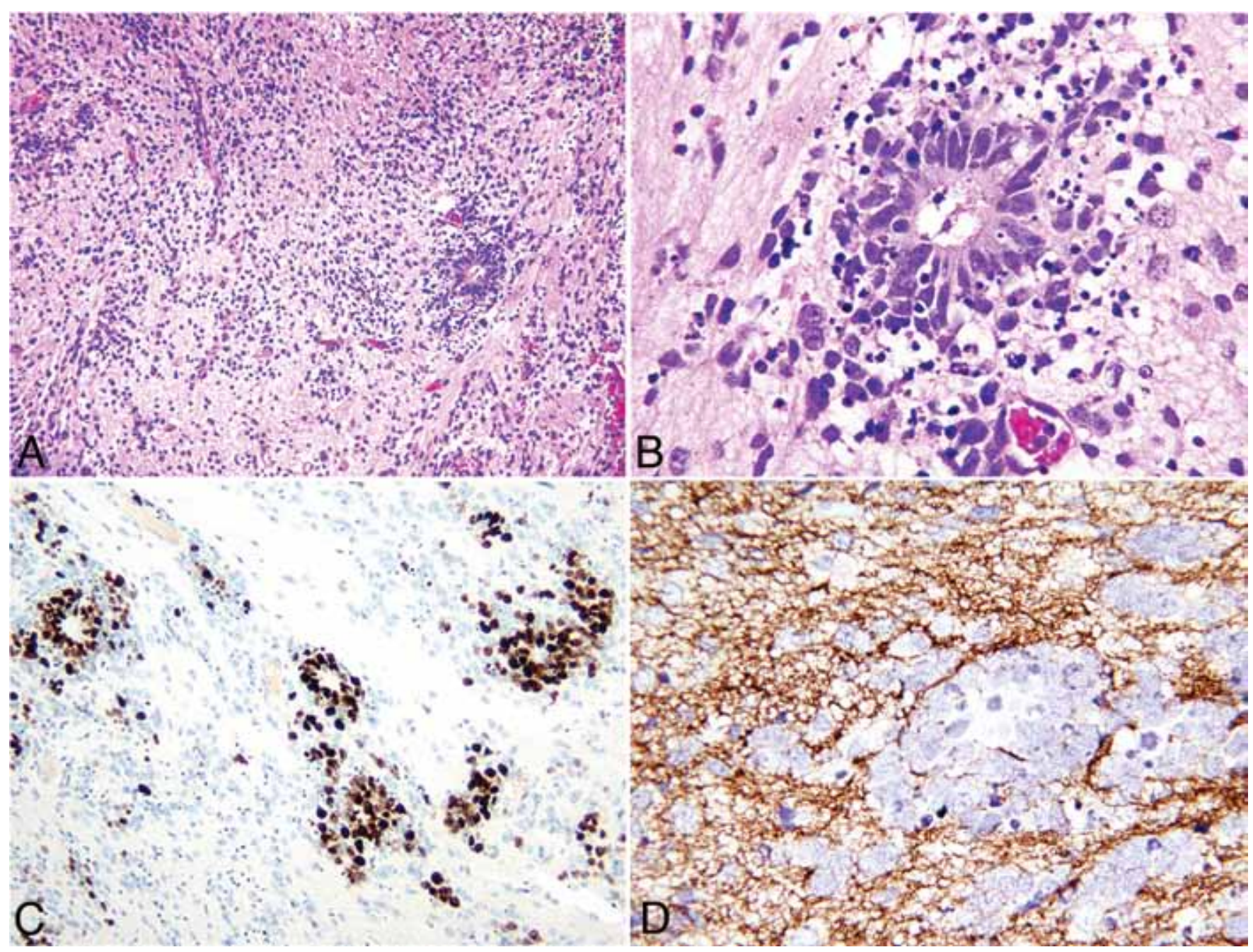

FIG. 2. Case 1. Histopathological features of ETANTR. A: Low-power photomicrograph demonstrating abundant neuropil containing occasional true rosettes. H \& E. B: High-power photomicrograph of true rosettes demonstrating multilayering of pleomorphic nuclei. H\&E. C: Low-power photomicrograph obtained after immunostaining for Ki 67, which highlights the proliferative activity of cells comprising the true rosettes. D: Photomicrograph obtained after immunostaining for synaptophysin. The neuropil separating the true rosettes is highlighted, but the cells comprising the rosettes are not stained. Original magnification $\times 100(\mathrm{~A}$ and $\mathrm{C}), \times 200(\mathrm{D})$, and $\times 400(\mathrm{~B})$.

tubules and tumor cells with neurosecretory granules suggested to them a neuroblastic nature of the tumor and accounted for the diffuse synaptophysin immunostain positivity. Occasional glial differentiation of the tumor explained the GFAP positivity. However, the name was subsequently changed to "embryonal tumor with abundant neuropil and true rosettes" (ETANTR) after other groups reported 17 similar cases. Subsequently, Eberhart

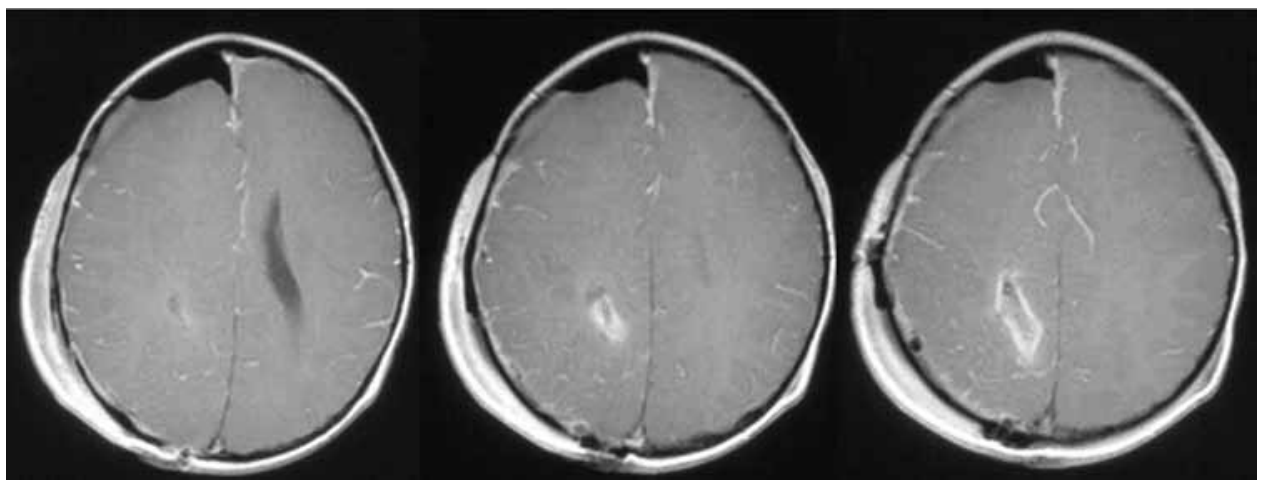

FIG. 3. Case 1. Immediate-postoperative Gd-enhanced MR images showing radical tumor resection. 


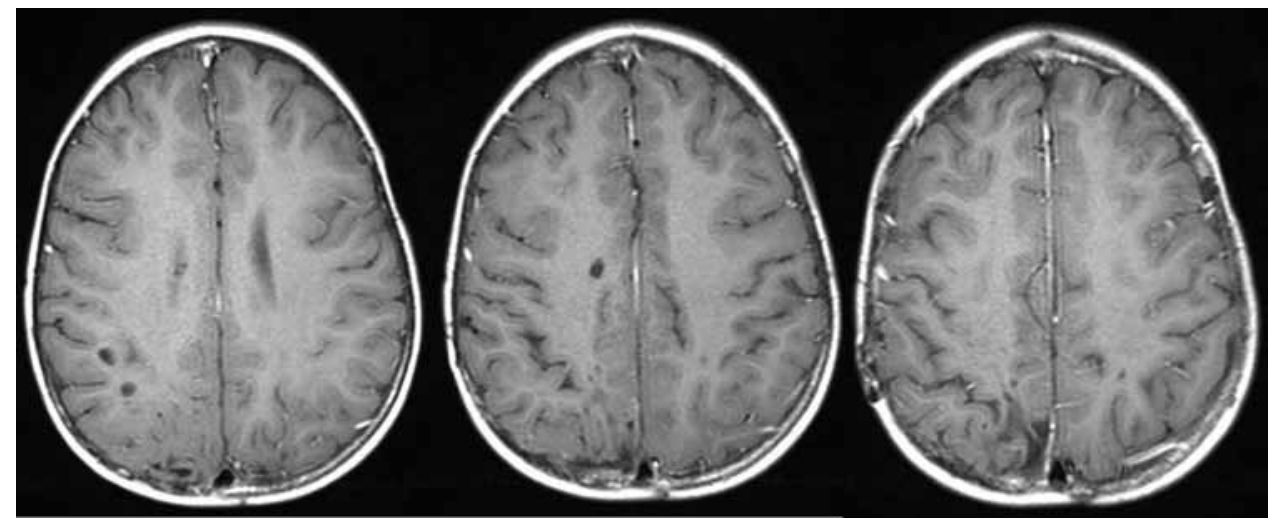

FIG. 4. Case 1. Follow-up Gd-enhanced MR images obtained 7 years after surgery showing absence of recurrent tumor.

and colleagues reported an additional 22 cases in 2009, thus making a total of 46 cases of ETANTR reported in the literature to date. . $, 3,5-9,11,13,15^{-15}$

ETANTR has been identified as a histologically distinctive CNS PNET. It is characterized by the presence of undifferentiated neuroepithelial cells resembling those of the classic PNET as well as broad zones of welldifferentiated neuropil and ependymoblastic rosettes arising abruptly from paucicellular regions of neoplastic neuropil. The rosette-forming cells form a multilayered (pseudostratified) wall in which both mitoses and karyorrhectic debris are seen, typically without a luminal basement membrane. Homer Wright rosettes (pathognomonic of neuroblastic differentiation in medulloblastoma) may also be present. A well-defined internal limiting membrane is a hallmark of these lesions, with the outer cells of the rosette merging into the adjacent neuropil and embryonal cells. ${ }^{9}$ Medulloblastoma, on the other hand, shows classical Homer-Wright rosettes, and medulloepithelioma shows a canalicular growth pattern with an intact luminal basement membrane and divergent differentiation. Medulloblastoma itself is a very heterogeneous neoplasm with several variants such as classic, desmoplastic, largecell anaplastic, melanotic, and medullomyoblastoma. These lesions show divergent neuronal differentiation with neuroblastic rosettes, epithelial, glial, and retinoblastoma-like differentiation., ${ }^{4,10,13}$ Anaplastic ependymomas, however, show perivascular pseudorosettes that carry a sharp distinction from true rosettes (Table 1). 4,10,16

The diagnosis of ependymoblastoma is controversial and has been debated since its initial description in 1926.
Recently this subcategory has been considered to be neither precise nor specific. ${ }^{11}$ Ependymoblastic rosettes, once considered the hallmark of ependymoblastoma, are no longer considered pathognomonic for this tumor. Judkins and Ellison ${ }^{11}$ reviewed 14 cases that had been diagnosed previously as ependymoblastoma and reclassified 8 of them as ETANTR on the basis of the embryonal nature of the cells. They noted that ependymoblastic rosettes were more frequently encountered in ETANTR than in other CNS embryonal neoplasms. They went on to suggest withdrawing the diagnosis of ependymoblastoma from the classification of CNS tumors.

In an effort to further distinguish ETANTR, some investigative groups have recently performed cytogenetic studies on pathological specimens. Fuller and colleagues ${ }^{8}$ found abnormalities on chromosome 17 in 2 cases (isochromosome $17 \mathrm{q}$ in 1 tumor and polysomy of 17 in the other), anomalies that are typical of medulloblastoma. This suggests that ETANTR, despite arising at locations outside the cerebellum, may be more closely related to cerebellar medulloblastomas than supratentorial PNETs. Subsequently, Gessi and colleagues ${ }^{9}$ found extra copies of chromosome 2 in both of the 2 cases in which they performed cytogenetic testing. Buccoliero and colleagues ${ }^{3}$ also found chromosome 2 polysomy in 1 case, as did Pfister and colleagues in 1 case. ${ }^{15}$ Although duplication of genetic material on chromosome 17 and loss of genetic material on chromosome 22 has been well documented in embryonal CNS tumors, chromosome 2 polysomy may be a differentiating feature of ETANTR. ${ }^{2}$

Although ETANTR shows some cytogenetic features

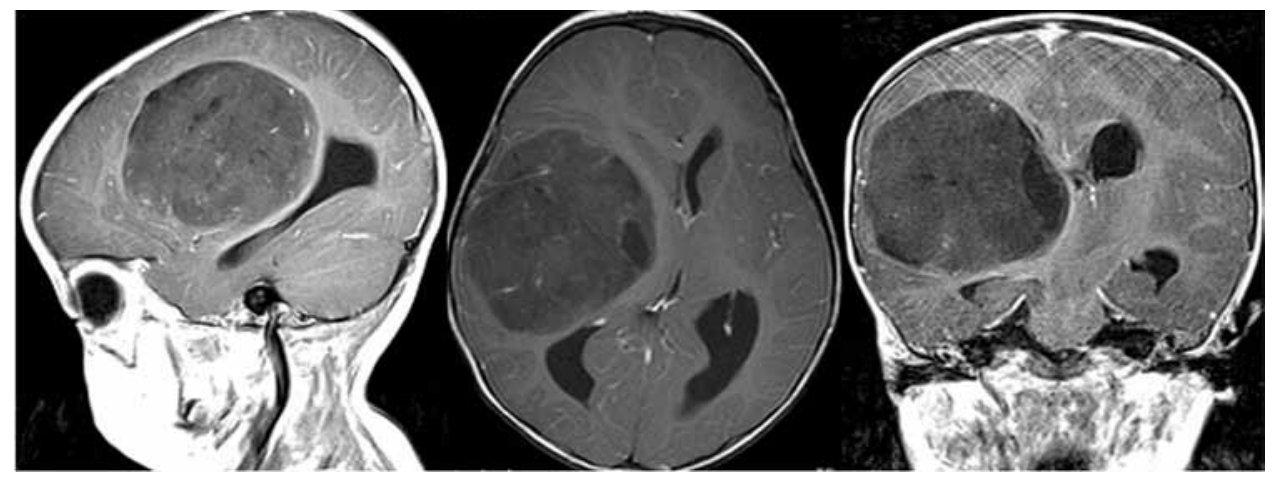

FIG. 5. Case 2. Preoperative Gd-enhanced sagittal, axial, and coronal MR images. 


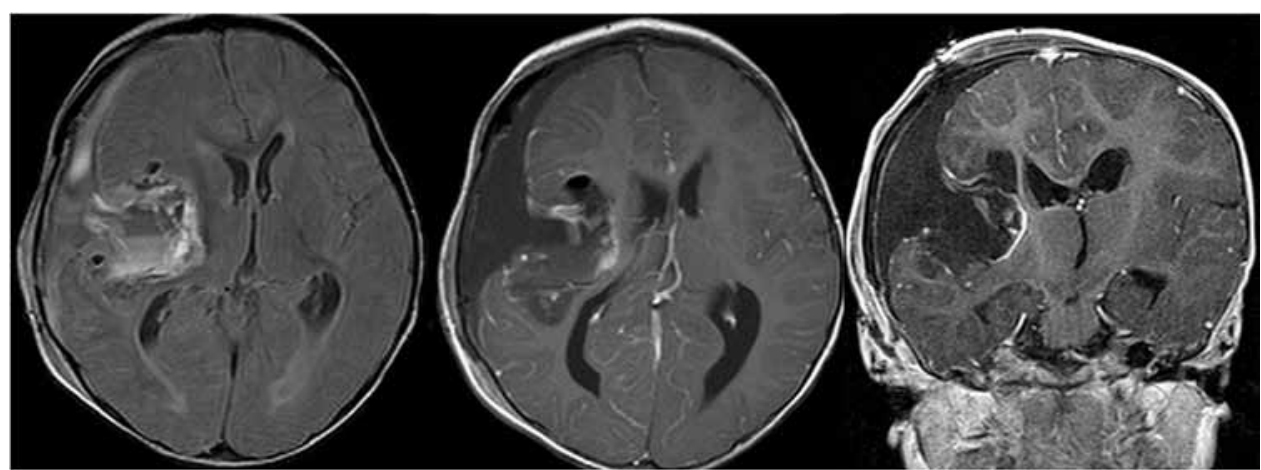

FIG. 6. Case 2. Immediate postoperative MR images showing radical resection.

resembling medulloblastoma, recent molecular studies have also demonstrated analogies between ETANTR and ependymoblastoma. Pfister and colleagues, ${ }^{15}$ who noted the chromosome 2 polysomy in ETANTR, also found a high-level amplification on chromosome 19 at position 19q13.42 causing an upregulation of microRNA clusters and several protein-coding genes. This molecular aberration had not been described in any other brain tumor, further supporting the hypothesis that ETANTR was a distinct tumor entity.

Subsequently, Korshunov and colleagues ${ }^{12}$ used fluorescence in situ hybridization to evaluate tumors that had been diagnosed morphologically as either ETANTR or ependymoblastoma. They demonstrated focal genomic amplification at 19q13.42 in 37 of 40 samples (93\%). Among tumors harboring this amplification, 19 samples were identified as ETANTR and 18 as ependymoblastoma. This suggests that ETANTR and ependymoblastoma may be closely related.

The natural history of ETANTR is similar to that of other malignant pediatric brain tumors. ETANTRs occur in the pediatric population, usually in children 4 years of age or younger, with a slight female preponderance compared with other PNETs. Although these neoplasms are found in supra- and infratentorial locations, there seems to be a predilection for the supratentorial space, especially the frontal and parietal lobes, where they tend to occur as large, cystic, sometimes calcific intraaxial masses. The radiological differential diagnosis of these lesions includes desmoplastic infantile ganglioglioma, PNET, and supratentorial ependymoma. Gessi and colleagues ${ }^{9}$ reported one case with leptomeningeal dissemination that responded to intrathecal chemotherapy. Infantile lesions, in general, are highly malignant and generally associated with a poor clinical outcome, despite aggressive surgical and adjuvant therapy. Like PNETs, these tumors appear to be less sensitive to chemotherapy. The patient in our illustrative Case 2, a 17-month-old child, fared poorly. He was treated with chemotherapy without radiation because of his young age. Both of our patients presented with a short history of rapid clinical deterioration over weeks, suggesting a fast-paced progression of this neoplasm.

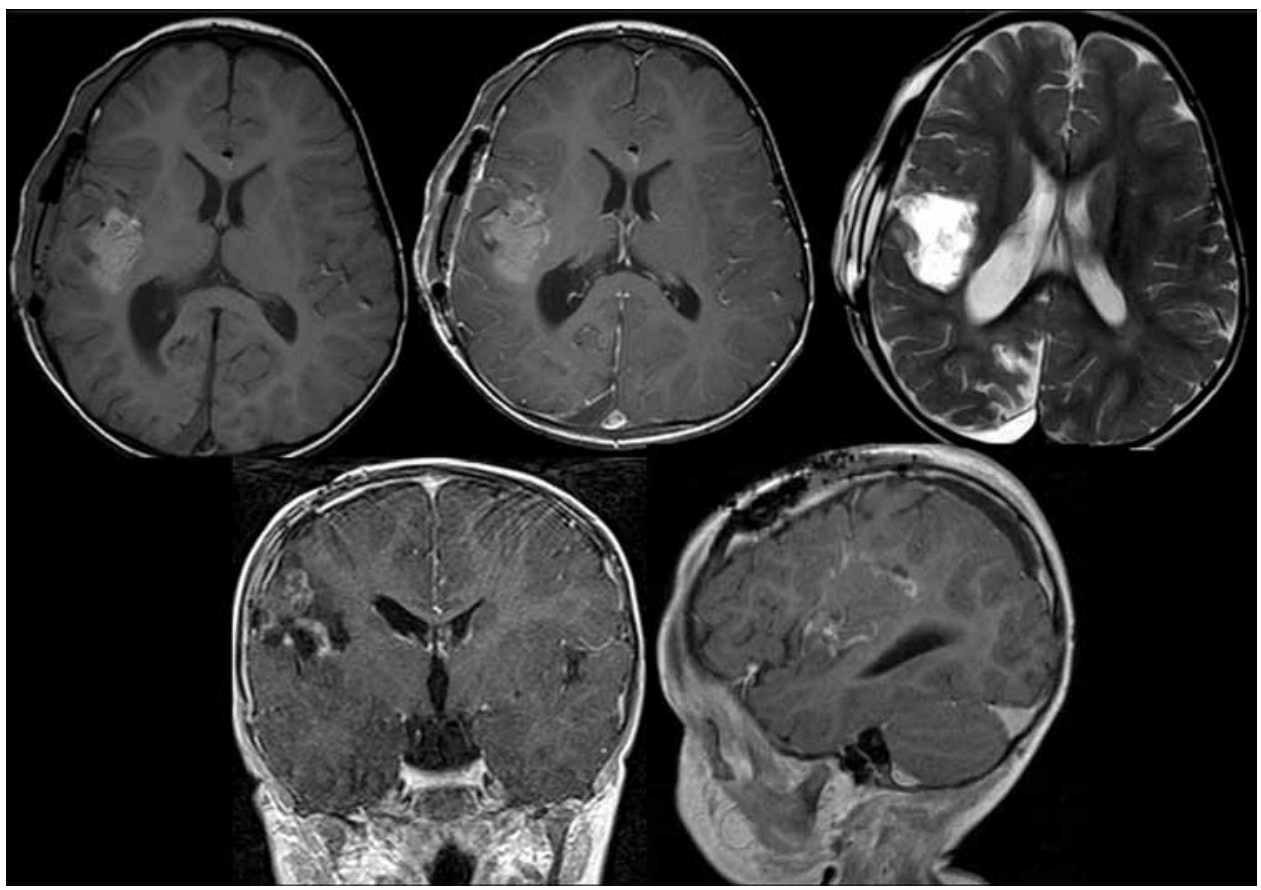

FIG. 7. Case 2. Follow-up MR images obtained 4 months after initial surgery showing tumor recurrence. 


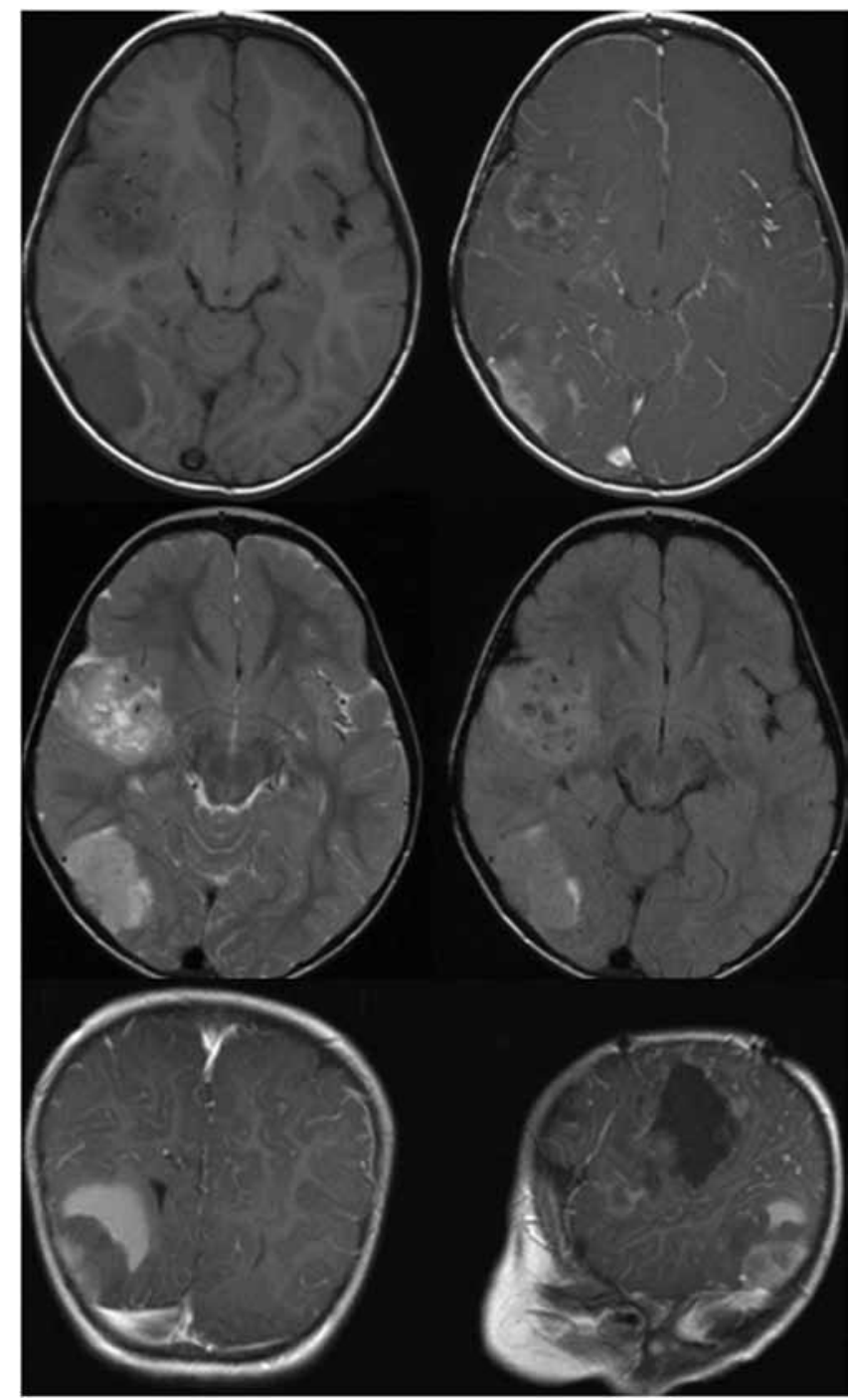

Fig. 8. Case 2. Follow-up MR images showing tumor recurrence after the second resection of the ETANTR, radiation, chemotherapy, and stem cell rescue.

Of the 46 cases of ETANTR reported to date, the clinical outcome has been poor, with the longest survival found to be only 42 months. The largest series was reported by Gessi and colleagues, ${ }^{9}$ who described 29 patients ( 7 cases from Eberhart and colleagues in 2000 and an additional 22 cases in 2009). Their patients ranged in age from 9 to 48 months. Older patients, who were treated with resection, chemotherapy, and radiation had a better outcome than younger infants, who had only resection and chemotherapy. The longest infant survival was 18 months. Al-Hussain and Dababo ${ }^{1}$ reported on a 2-year-old girl with a vermian ETANTR tumor. She underwent near-total resection followed by chemotherapy but developed a recurrence in 6 months. In 2010, Buccoliero and colleagues ${ }^{3}$ described a 2-year-old girl with a bihemispheric lesion of the parietooccipital lobes that was completely removed in 2 stages. In 2007, Dunham and colleagues ${ }^{5}$ reported on a 2 -year-old boy with a partly calcified cystic left temporoparietal hemispheric ETANTR who underwent near-total resection with no adjuvant therapy. He developed a poste-
TABLE 1: Histological differential diagnosis of ETANTR and characteristic features

\begin{tabular}{lc}
\hline \multicolumn{1}{c}{ Tumor Type } & \multicolumn{1}{c}{ Histological Characteristics } \\
\hline ETANTR & $\begin{array}{c}\text { multilayered true rosettes lacking a luminal } \\
\text { basement membrane } \\
\text { Homer-Wright rosettes } \\
\text { medulloblastoma } \\
\text { medulloepithelioma }\end{array}$ \\
$\begin{array}{c}\text { canalicular growth pattern; luminal base- } \\
\text { ment membrane; divergent differentiation } \\
\text { anaplastic ependymoma } \\
\text { perivascular pseudorosettes; monolayered } \\
\text { true rosettes }\end{array}$ \\
\hline
\end{tabular}

rior fossa tumor recurrence after 11 months necessitating urgent debulking. The parents declined further treatment. In 2006, Fuller and colleagues ${ }^{8}$ reported on 2 patients, a boy and girl, each 4 months old. The boy had a minimally enhancing, large, midpontine lesion that was debulked and subsequently treated with radiation and chemotherapy. He had stable disease at 19 months' follow-up. The girl, who had a left parietooccipital lesion, underwent 3 debulking procedures followed by high-dose chemotherapy and radiation but died within 6 months of the initial diagnosis. In 2006, La Spina and colleagues ${ }^{13}$ reported on a 4-year-old boy with a nonenhancing partly cystic intrinsic brainstem ETANTR. Following resection, he underwent hyperfractionated craniospinal radiation and aggressive multiagent chemotherapy and was alive and disease-free 34 months after surgery. In 2009, Pfister and colleagues ${ }^{15}$ described a 2-year-old girl with a vermian mass who underwent subtotal resection followed by chemotherapy, stem cell transplantation, and radiation therapy, with stabilization of disease at 16 months follow-up. In 2010, Ferri Niguez and colleagues ${ }^{7}$ reported on 2 girls aged 9 and 23 months who both underwent resection and chemotherapy. The latter patient received radiation therapy also. However, both died after 10 months.

\section{Conclusions}

ETANTR has been identified as a histologically distinctive, PNET of the CNS. Ependymoblastic rosettes, previously felt to be diagnostic of ependymoblastoma, are more frequently seen in ETANTR than other embryonal tumors. Since the initial description of ETANTR in 2000, the limited reports in the literature have shown a female predominance. The patients are usually young children, presenting before the age of 4 years. ETANTR has unique cytogenetic features including focal genomic amplification of $19 \mathrm{q} 13.42$ as well as chromosome 2 polysomy. The treatment strategy is similar to that of PNETs, including resection, systemic chemotherapy, and craniospinal radiation when possible. Although this tumor has a high potential for malignancy and has been associated with a dismal prognosis, it appears that long-term survival is possible following radical surgery and adjuvant therapy, as we have reported in Case 1. As we learn more about the molecular and genetic basis of this malignant neoplasm, it may be possible to develop targeted and more effective therapies. 


\section{Disclosure}

The authors report no conflict of interest concerning the materials or methods used in this study or the findings specified in this paper.

Author contributions to the study and manuscript preparation include the following. Conception and design: Manjila. Acquisition of data: Manjila, Ray, Hu, Cai, ML Cohen. Analysis and interpretation of data: Manjila, Ray, ML Cohen. Drafting the article: AR Cohen, Manjila, Ray, ML Cohen. Critically revising the article: AR Cohen, Manjila, Ray, ML Cohen. Reviewed final version of the manuscript and approved it for submission: AR Cohen, Manjila. Administrative/technical/material support: Ray, Hu, Cai, ML Cohen. Study supervision: AR Cohen, Hu, ML Cohen.

\section{Acknowledgments}

The authors are grateful to Dr. Peter C. Burger of Johns Hopkins Medical Center for his review of the pathological specimens and confirmation of the diagnosis in Case 1. They also thank Dr. Patricia Robertson of Mott Children's Hospital, University of Michigan, who managed the adjuvant therapy for Case 1 .

\section{References}

1. Al-Hussain TO, Dababo MA: Posterior fossa tumor in a 2 year-old girl. Brain Pathol 19:343-346, 2009

2. Biegel JA, Allen CS, Kawasaki K, Shimizu N, Budarf ML, Bell CJ: Narrowing the critical region for a rhabdoid tumor locus in 22q11. Genes Chromosomes Cancer 16:94-105, 1996

3. Buccoliero AM, Castiglione F, Degl'Innocenti DR, Franchi A, Paglierani M, Sanzo M, et al: Embryonal tumor with abundant neuropil and true rosettes: morphological, immunohistochemical, ultrastructural and molecular study of a case showing features of medulloepithelioma and areas of mesenchymal and epithelial differentiation. Neuropathology 30:84-91, 2010

4. Burger PC, Grahmann FC, Bliestle A, Kleihues P: Differentiation in the medulloblastoma. A histological and immunohistochemical study. Acta Neuropathol 73:115-123, 1987

5. Dunham C, Sugo E, Tobias V, Wills E, Perry A: Embryonal tumor with abundant neuropil and true rosettes (ETANTR): report of a case with prominent neurocytic differentiation. J Neurooncol 84:91-98, 2007

6. Eberhart CG, Brat DJ, Cohen KJ, Burger PC: Pediatric neuroblastic brain tumors containing abundant neuropil and true rosettes. Pediatr Dev Pathol 3:346-352, 2000

7. Ferri Niguez B, Martínez-Lage JF, Almagro MJ, Fuster JL,
Serrano C, Torroba MA, et al: Embryonal tumor with abundant neuropil and true rosettes (ETANTR): a new distinctive variety of pediatric PNET: a case-based update. Childs Nerv Syst 26:1003-1008, 2010

8. Fuller C, Fouladi M, Gajjar A, Dalton J, Sanford RA, Helton KJ: Chromosome 17 abnormalities in pediatric neuroblastic tumor with abundant neuropil and true rosettes. Am J Clin Pathol 126:277-283, 2006

9. Gessi M, Giangaspero F, Lauriola L, Gardiman M, Scheithauer BW, Halliday W, et al: Embryonal tumors with abundant neuropil and true rosettes: a distinctive CNS primitive neuroectodermal tumor. Am J Surg Pathol 33:211-217, 2009

10. Jaffey PB, To GT, Xu HJ, Hu SX, Benedict WF, Donoso LA, et al: Retinoblastoma-like phenotype expressed in medulloblastomas. J Neuropathol Exp Neurol 54:664-672, 1995

11. Judkins AR, Ellison DW: Ependymoblastoma: dear, damned, distracting diagnosis, farewell! Brain Pathol 20:133-139, 2010

12. Korshunov A, Remke M, Gessi M, Ryzhova M, Hielscher T, Witt H, et al: Focal genomic amplification at 19q13.42 comprises a powerful diagnostic marker for embryonal tumors with ependymoblastic rosettes. Acta Neuropathol 120:253260, 2010

13. La Spina M, Pizzolitto S, Skrap M, Nocerino A, Russo G, Di Cataldo A, et al: Embryonal tumor with abundant neuropil and true rosettes. A new entity or only variations of a parent neoplasms (PNETs)? This is the dilemma. J Neurooncol 78: 317-320, 2006

14. Louis DN, Ohgaki H, Wiestler OD, Cavenee WK, Burger PC, Jouvet A, et al: The 2007 WHO classification of tumours of the central nervous system. Acta Neuropathol 114:97-109, 2007

15. Pfister S, Remke M, Castoldi M, Bai AH, Muckenthaler MU, Kulozik A, et al: Novel genomic amplification targeting the microRNA cluster at $19 \mathrm{q} 13.42$ in a pediatric embryonal tumor with abundant neuropil and true rosettes. Acta Neuropathol 117:457-464, 2009

16. Sugita Y, Tokunaga O, Terasaki M, Morimatsu M, Shigemori M: Epithelial differentiation in medulloblastoma: comparison with other embryonal tumors of neuroectodermal origin. Pathol Int 53:858-864, 2003

Manuscript submitted September 15, 2010.

Accepted October 13, 2010.

Address correspondence to: Alan R. Cohen, M.D., Division of Pediatric Neurosurgery, Rainbow Babies and Children's Hospital, 11100 Euclid Avenue, Basement-501, Cleveland, Ohio 44106. email: alan.cohen@uhhs.com. 